\title{
Óxido Nítrico Inalatório para Crianças com Síndrome do Desconforto Respiratório Agudo*
}

\section{Inhaled Nitric Oxide for Children with Acute Respiratory Distress Syndrome}

\author{
José Roberto Fioretto ${ }^{1}$, Mário Ferreira Carpi ${ }^{2}$, Rossano César Bonatto ${ }^{3}$, \\ Sandra Mara Queiroz Ricchetti ${ }^{4}$, Marcos Aurélio de Moraes ${ }^{4}$.
}

\section{RESUMO}

JUSTIFICATIVA E OBJETIVOS: O objetivo desse estudo foi rever a literatura sobre a utilização de óxido nítrico inalatório em crianças com síndrome do desconforto respiratório agudo.

CONTEÚDO: Revisão bibliográfica e seleção de publicações mais relevantes sobre óxido nítrico inalatório, utilizando a base de dados MedLine e Cochrane de Revisões Sistemáticas. A revisão incluiu descrição de aspectos da definição, fisiopatologia e tratamento ventilatório da síndrome do desconforto respiratório agudo, assim como o metabolismo, efeitos biológicos e aplicação clínica do óxido nítrico inalatório, comentando dose, administração e retirada do gás, precações, efeitos adversos e contra-indicações.

CONCLUSÕES: O óxido nítrico, vasodilatador pulmonar

1. Livre Docente da Disciplina de Medicina Intensiva Pediátrica do Departamento de Pediatria da FMB - UNESP; Chefe da Unidade de Terapia Intensiva Pediátrica da UNESP.

2. Professor Assistente Doutor do Departamento de Pediatria da FMB - UNESP; Médico Diarista da Unidade de Terapia Intensiva Pediátrica da UNESP.

3. Professor Assistente Doutor do Departamento de Pediatria da FMB - UNESP; Médico Diarista da Unidade de Terapia Intensiva Pediátrica da UNESP.

4. Médicos Diaristas da Unidade de Terapia Intensiva Pediátrica da UNESP.

*Recebido da UTI-Pediátrica do Departamento de Pediatria da Faculdade de Medicina de Botucatu - Universidade Estadual Paulista (FMB - UNESP), Botucatu, SP

Apresentado em 13 de outubro de 2006

Aceito para publicação em 27 de novembro de 2006

Endereço para correspondência:

Prof. Adjunto Dr. José Roberto Fioretto

Departamento de Pediatria da UNESP

18.618-970 Botucatu, SP

Fone: (14) 3811-6274.

E-mail: jrf@fmb.unesp.br

(C)Associação de Medicina Intensiva Brasileira, 2006 seletivo, tem efeitos benéficos sobre as trocas gasosas e ventilação em crianças com hipóxia grave. É seguro quando administrado em ambiente de tratamento intensivo sob rigorosa monitorização. Estudos aleatórios e controlados devem enfocar a administração precoce do gás na síndrome do desconforto respiratório agudo, quando essa é potencialmente reversível.

Unitermos: óxido nítrico inalatório, Pediatria, síndrome do desconforto respiratório agudo, ventilação mecânica

\section{SUMMARY}

BACKGROUND AND OBJECTIVE: The objective of this study was to review the literature on inhaled nitric oxide to children with acute respiratory distress syndrome.

CONTENTS: A review of literature and selection of the most important publications on inhaled nitric oxide, using the MedLine and Cochrane Systematic Review Databases. This review was organized as follows: introduction; metabolism and biological effects; clinical applications; dosage, gas administration and weaning process; warnings and side-effects. Inhaled nitric oxide use was described in acute respiratory distress syndrome.

CONCLUSIONS: Inhaled nitric oxide as the first vasodilator to produce selective pulmonary vasodilation has beneficial effects on gas exchange and ventilation, improving outcome in children with severe hypoxia. It is safe when administered in intensive care units under strict surveillance and monitoring. Further studies should be concentrated on early treatment, when acute respiratory distress syndrome is potentially reversible.

Key Words: acute respiratory distress syndrome, inhaled nitric oxide, mechanical ventilation, Pediatric.

\section{INTRODUÇÃO}

A síndrome do desconforto respiratório agudo (SDRA) foi inicialmente descrita por Ashbaugh e col. ${ }^{1}$ em uma 
série de pacientes que desenvolveram falência respiratória aguda grave. Atualmente, apesar do melhor entendimento da fisiopatologia e do avanço tecnológico observado na monitorização e no tratamento de pacientes com SDRA, a mortalidade permanece elevada ${ }^{2}$.

Em 1994, a Conferência de Consenso Americana-Européia ${ }^{3}$ estabeleceu os critérios diagnósticos da síndrome, sendo eles: 1) evento agudo desencadeante de lesão pulmonar; 2) hipoxemia refratária à administração de oxigênio demonstrada por relação $\mathrm{PaO}_{2} / \mathrm{FiO}_{2}<$ $200 \mathrm{mmHg}$, a despeito da PEEP utilizada; 3) infiltrado bilateral na radiografia de tórax em posição frontal e 4) $P C P \leq 18 \mathrm{mmHg}$, quando medida, ou ausência de evidência clínica de hipertensão atrial esquerda.

A SDRA pode ser causada por agressão pulmonar primária ou secundária. É caracterizada por processo inflamatório que leva à quebra da barreira alvéolo-capilar com desenvolvimento de edema intersticial e alveolar, diminuição da complacência pulmonar, desequilíbrio da relação ventilação/perfusão (V/Q) e hipoxemia refratária à administração de oxigênio. Além disso, existe aumento da resistência vascular pulmonar produzido por uma combinação complexa de lesão inflamatória pulmonar primária e agressividade da ventilação mecânica (VM). A hipertensão pulmonar impõe carga adicional ao ventrículo direito, limitando o débito cardíaco ${ }^{4}$.

\section{TRATAMENTO VENTILATÓRIO DA SDRA}

A VM constitui um dos pilares do tratamento da SDRA na medida em que melhora a oxigenação por recrutamento alveolar, com restabelecimento da relação V/Q. Estudo tomográfico de pulmões de pacientes com SDRA contribuiu decisivamente para o entendimento da doença. Foi demonstrado que o comprometimento do parênquima pulmonar não é homogêneo, havendo áreas pulmonares com relação $\mathrm{V} / \mathrm{Q}$ relativamente normais, coexistindo com áreas totalmente consolidadas e regiões hiperdistendidas ${ }^{5}$. Paralelamente, foi demonstrado que a VM pode lesar as áreas de pulmão normal ou aumentar a lesão daquelas já comprometidas, introduzindo o conceito de lesão pulmonar induzida pela VM (LPIVM) ${ }^{6-8}$. Na SDRA, extensas áreas alveolares tornam-se consolidadas com diminuição de alvéolos disponíveis para as trocas gasosas, tornando o pulmão funcionalmente análogo ao baby lung, quanto ao seu tamanho. O emprego de volume corrente (VC) elevado dentro deste "pequeno" pulmão pode levar a hiperdistensão dos alvéolos que estão abertos ${ }^{9}$.
Atualmente, existem evidências de que o estiramento alveolar induzido por VC elevado ou por elevadas pressões utilizadas no ventilador desempenha papel importante na gênese da LPIVM, pois determina hiperdistensão alveolar nas áreas normais e reabertura cíclica de alvéolos colapsados com conseqüente lesão inflamatória estrutural de áreas pulmonares bem ventiladas ${ }^{10-13}$.

A partir deste conhecimento, foi introduzida a estratégia ventilatória chamada de ventilação mecânica protetora, utilizada em conjunto com a estratégia para manter os alvéolos abertos (open lung approach). Estes métodos objetivam proteger o pulmão contra a lesão induzida pela VM, limitando o VC $(<6 \mathrm{~mL} / \mathrm{kg})$, ofertando nível adequado de PEEP (acima do ponto de inflexão inferior na curva estática pressão-volume) com pressão de platô $<20 \mathrm{mmHg}$ acima da PEEP, permitindo hipercapnia e hipoxemia $\left(\mathrm{SaO}_{2}\right.$ entre $88 \%$ e $\left.90 \%\right)$ e utilizando preferencialmente modos ventilatórios limitados a pressão.

O valor clínico da VM protetora em adultos, com SDRA, foi primeiramente demonstrado por Amato e col. ${ }^{14}$. Foi observada redução significante da taxa de mortalidade dos pacientes tratados com VC menor do que $6 \mathrm{~mL} /$ $\mathrm{kg}$ e pressão de platô de menos de $20 \mathrm{mmHg}$ acima da PEEP na comparação com pacientes tratados com VM convencional controlada a volume. Estes resultados foram posteriormente confirmados pelo estudo da ARDS Network ${ }^{15}$, que avaliou 861 adultos e demonstrou redução absoluta de $9 \%$ na mortalidade com VC mais baixo $(\leq 6 \mathrm{~mL} / \mathrm{kg})$ comparado com o tradicional (12 mL/kg). Estudo semelhante ainda não foi realizado em crianças. Entretanto, a dramática modificação do prognóstico de adultos com SDRA, demonstrada por estes estudos, provocaram importante impacto no tratamento da falência respiratória de crianças. O pensamento atual é que o emprego de VC baixo é provavelmente melhor também em crianças.

\section{ÓXIDO NÍTRICO INALATÓRIO NA SDRA}

A VM protetora não é destituída de problemas. O uso de baixo VC/pico de pressão inspiratória pode diminuir o volume pulmonar, com colapso alveolar e hipoxemia, os quais, por sua vez, podem aumentar a resistência vascular pulmonar ${ }^{16,17}$.

Levando-se em consideração a fisiopatologia da SDRA e as possíveis complicações da VM protetora, entende-se que a administração de fármacos vasodilatadores seria útil. No entanto, os vasodilatadores disponí- 
veis, administrados por via venosa, não apresentam especificidade para o leito vascular pulmonar e podem reduzir a pressão arterial sistêmica, agravando ainda mais o shunt intrapulmonar por vasodilatação não seletiva de artérias que perfundem áreas pulmonares mal ventiladas, com piora da relação $V / Q$ nestas áreas e diminuição da $\mathrm{PaO}_{2}{ }^{18}$.

No final de 1980, o óxido nítrico (NO) foi identificado como fator de relaxamento derivado do endotélio ${ }^{19}$. O NO é uma combinação 1:1 de dois dos mais abundantes gases da atmosfera, sendo sintetizado a partir da L-arginina pela ação da NO sintase e liberado após ativação química e mecânica. Quando produzido nas células vasculares endoteliais, o gás difunde-se para as células musculares lisas adjacentes e ativa a guanilciclase, que determina aumento da produção do GMP cíclico (guanosine 3,5'-monophosphate). O aumento do GMP cíclico diminui a concentração de cálcio intracelular com relaxamento da musculatura lisa e vasodilatação ${ }^{20}$.

O óxido nítrico inalatório (NOi) exibe efeitos macro e microsseletivos sobre a vasculatura pulmonar. O efeito macrosseletivo é obtido por meio de vasodilatação direta das artérias pulmonares e o microsseletivo deve-se à sua ação limitada às regiões pulmonares bem ventiladas. Esta vasodilatação seletiva direciona o fluxo sangüíneo de áreas mal ventiladas para áreas bem ventiladas e com perfusão diminuída, melhorando a relação $V / Q$ e a oxigenação, com diminuição da resistência vascular pulmonar e da pós-carga do ventrículo direito. Pela otimização da relação $\mathrm{V} / \mathrm{Q}$, o gás pode permitir o emprego de VM menos agressiva, que, por sua vez, pode diminuir o risco de LPIVM e a morbidade. Além disso, os efeitos locais do NOi sobre a inflamação, edema e permeabilidade capilar o tornam atraente para ser utilizado em insuficiência respiratória aguda grave. Destaca-se que o gás é inativado rápida e especificamente no sangue, pela alta afinidade pela hemoglobina, não promovendo vasodilatação sistêmica ${ }^{21,22}$.

No começo de 1990, os efeitos benéficos do NOi sobre a oxigenação, hipertensão pulmonar e índice cardíaco foram descritos em adultos ${ }^{23}$ e crianças ${ }^{24}$ com SDRA. Posteriormente, vários estudos, enquanto confirmaram os efeitos imediatos, não demonstraram resposta sustentada a terapia com NOi em adultos ${ }^{25,26}$ ou crian$\operatorname{ças}^{27,28}$. No entanto, Dobyns e col. ${ }^{29}$, em estudo aleatório e controlado, observaram resposta sustentada ao NOi versus tratamento com placebo em subgrupos de crianças (aquelas com índice de oxigenação $\geq 25$ e as imunodeprimidas). Os autores admitiram que o tratamento com NOi não sustentou a melhora da oxigenação em todos os pacientes pois eles foram incluídos em estágios tardios da doença.

A hipótese de que a resposta ao NOi depende do momento de sua introdução foi relatada por outros autores e testada em estudo experimental que demonstrou que a exposição precoce ao gás, no curso da lesão pulmonar aguda induzida por sepse, associa-se à redução da infiltração leucocitária e menor lesão oxidativa pulmonar ${ }^{30}$.

Seguindo a idéia dos efeitos benéficos da administração precoce, Fioretto e col. publicaram, em 2001, um protocolo de administração precoce de NOi em crianças com SDRA. Demonstrou-se melhora imediata e sustentada da oxigenação utilizando o NOi tão precocemente quanto 12 horas depois do diagnóstico da doença, suportando a hipótese de que o tratamento precoce com o gás pode ser mais efetivo ${ }^{31}$.

Em 2003, The Cochrane Library publicou revisão sistemática sobre os efeitos do NOi em falência respiratória hipoxêmica aguda de adultos e crianças, com atualização em 2006 ${ }^{32}$. Cinco estudos aleatórios e controlados foram analisados, avaliando 535 pacientes. A revisão concluiu que o NOi é útil apenas como terapia de salvamento, nas primeiras 24 horas de doença. Vale ressaltar que dos cinco estudos analisados, apenas o de Dobyns e col. foi realizado em crianças $^{29}$ e que a conclusão final indicou que o potencial efeito do tratamento com NOi em pacientes com SDRA está para ser definido, principalmente em crianças.

Em 2004, Fioretto e col..$^{33}$ analisaram os efeitos da administração precoce de NOi na SDRA comparando um grupo composto de crianças seguidas prospectivamente e que recebeu NOi associado ao tratamento convencional (GNOi) com um grupo histórico de pacientes que utilizou apenas o tratamento convencional (GTC). O objetivo central deste estudo foi determinar o efeito imediato e sustentado do NOi sobre alguns índices de oxigenação e parâmetros ventilatórios. O tratamento com NOi foi introduzido tão cedo quanto 1,5 horas depois do diagnóstico de SDRA, em pacientes otimamente ventilados com níveis apropriados de PEEP ( $\geq 10 \mathrm{cmH}_{2} \mathrm{O}$ ). Demonstrou-se melhora imediata da relação $\mathrm{PaO}_{2} / \mathrm{FiO}_{2}$ e do índice de oxigenação. $\mathrm{Da}$ mesma forma, o tratamento prolongado associou-se com melhora da oxigenação, tanto que a $\mathrm{FiO}_{2}$ e a Pip puderam ser rápida e significantemente reduzidas. $\mathrm{A}$ taxa de mortalidade para o GNOi foi mais baixa (GTC: 
10/21- 47,6\%; GNOi: 3/18 - 16,6\%, p < 0,001). A mediana do tempo de tratamento com o gás foi de 2 dias $(1-6)$ e a dose média utilizada de 4,03 $\pm 1,59 \mathrm{ppm}$. Não houve efeitos adversos durante a administração do gás. A retirada do NOi causou um "rebote" da hipoxemia em duas crianças, rapidamente corrigida com a reinstalação do tratamento. Concluiu-se que o tratamento precoce com NOi causa melhora imediata e sustentada da oxigenação, com redução mais precoce de parâmetros ventilatórios associados com alto risco de LPIVM e toxicidade pelo oxigênio (Pip e $\mathrm{FiO}_{2}$ ), o que pode contribuir para redução da taxa de mortalidade. Para testar esta hipótese são necessários ensaios clínicos aleatórios e controlados que enfoquem a administração precoce do NOi em crianças com SDRA.

Atualmente, o NOi é parte integrante do protocolo de tratamento desta Instituição. O gás é administrado assim que o diagnóstico de SDRA é estabelecido, na dose de 5 ppm, para pacientes com $\mathrm{SaO}_{2}<88 \%$, apesar de uma PEEP $\geq 10 \mathrm{cmH}_{2} \mathrm{O}$ e $\mathrm{FiO}_{2} \geq 0,6$.

Muitos estudos não demonstraram impacto do tratamento com NOi sobre a mortalidade. No entanto, é preciso considerar que existem diferentes fatores que interferem com a resposta prolongada de pacientes com SDRA ao NOi, tais como dose do gás, diferenças entre os pacientes, gravidade da doença de base e se a SDRA é primária ou secundária. Além disso, já se sabre que a resposta ao NOi é fortemente influenciada pela aplicação de uma PEEP adequa$\mathrm{da}^{34,35}$. De acordo com recomendações recentes ${ }^{36-38}$, o uso clínico do NOi na SDRA deve limitar-se a pacientes otimamente ventilados com PEEP, para que haja recrutamento de alvéolos adicionais para a troca gasosa e difusão do gás.

Em vista da complexidade fisiopatológica da SDRA, será muito difícil encontrar uma única forma de tratamento que, isoladamente, resolva o dilema terapêutico da síndrome. Ao contrário, o NOi deve ser utilizado em conjunto com outras formas de tratamento tais como posição prona, ventilação oscilatória de alta freqüência, manobras de recrutamento alveolar e ventilação mecânica protetora, as quais parecem ter efeito sinérgico com o gás ${ }^{39,40}$.

\section{ADMINISTRAÇÃO DE NOi: ASPECTOS TÉCNICOS E TOXICIDADE}

A administração de NOi segue protocolo estabelecido que deve ser aplicado sob rigorosa monitorização ${ }^{33,37}$.
Resumidamente, uma fração de NOi é continuamente liberada, via fluxômetro, diretamente dentro do ramo inspiratório do circuito do ventilador, distalmente ao umidificador, a $30 \mathrm{~cm}$ do tubo traqueal. As concentrações de NOi e de dióxido de nitrogênio $\left(\mathrm{NO}_{2}\right)$ são continuamente medidas por meio de sensor eletroquímico ou de quimioluminescência a partir de amostras de gás obtidas o mais perto possível do tubo traqueal. $\mathrm{O}$ alarme audiovisual deve ser mantido em um ppm acima da dose de NOi administrada e no nível máximo de $\mathrm{NO}_{2}$ de 3 ppm.

A inalação de NO parece ser segura, sendo os principais problemas a formação de $\mathrm{NO}_{2}$, metemoglobina e o efeito rebote.

$\mathrm{O} \mathrm{NO}_{2}$ é produzido a partir do $\mathrm{NO}$ e oxigênio, contaminando $\mathrm{o}$ ar ambiente e produzindo lesão oxidativa em bronquíolos terminais e alvéolos proximais. A taxa de produção de $\mathrm{NO}_{2}$ depende da dose de $\mathrm{NOi}, \mathrm{FiO}_{2}$ utilizada e duração do tratamento com o gás, sendo a quantidade de $\mathrm{NO}_{2}$ formado $1,14 \%$ da dose de NOi. Em uma simulação utilizando um modelo de pulmão e ventiladores comercialmente disponíveis, a produção de $\mathrm{NO}_{2}$ durante inalação com 20 ppm de $\mathrm{NO}$ parece ser mínima $\left(<0,7\right.$ ppm), mesmo com $\mathrm{FiO}_{2}$ tão alta quanto $95 \%{ }^{41}$.

A reação do NO com a hemoglobina produz metemoglobina. Níveis de metemoglobina $2 \%$ acima do total de hemoglobina podem prejudicar a liberação do oxigênio e piorar a hipóxia tecidual. Os níveis de metemoglobina devem ser avaliados antes de começar a administração do gás, depois de uma hora e a qualquer aumento da dose. Após estabilização, a monitorização pode ser diária.

O fenômeno do rebote é a piora da oxigenação e a hipertensão pulmonar que ocorre depois da retirada abrupta do gás ${ }^{31,42}$. Tal fenômeno pode ser explicado pelo fato de que o NO exógeno pode inibir de forma reversível a NO sintase presente nas vias aéreas e na circulação pulmonar e diminuir a produção pulmonar endógena do gás. É fundamental elaborar protocolo para a retirada lenta do gás e evitar interrupção acidental ou falhas na administração.

As contra-indicações ao uso de NOi podem ser absolutas e relativas. Entre as absolutas são descritos o déficit de redutase de metemoglobina e a utilização em recém-nascidos sabidamente dependentes de shunt sanguíneo da direta para a esquerda. Entre as relativas, citam-se quadros de diátese hemorrágica, hemorragia intracraniana e falência cardíaca esquerda grave 22,31 . 


\section{CONCLUSÃO}

O óxido nítrico, vasodilatador pulmonar seletivo, tem efeitos benéficos sobre as trocas gasosas e ventilação em crianças com hipóxia grave. É seguro quando administrado em ambiente de terapia intensiva sob rigorosa monitorização. Estudos controlados que enfoquem a administração precoce do gás são necessários em muitas condições, principalmente na SDRA.

\section{REFERÊNCIAS}

01. Ashbaugh DG, Bigelow DB, Petty TL et al - Acute respiratory distress in adults. Lancet, 1967;12:319-323.

02. Fioretto JR, Ferrari GF, Ricchetti SMQ et al - Síndrome do desconforto respiratório agudo em crianças: incidência, mortalidade e trocas gasosas. RBTI, 2001;2:58-62.

03. Bernard GR, Artigas A, Brigham KL et al - Report of the AmericanEuropean consensus conference on ARDS: definitions, mechanisms, relevant outcomes and clinical trial coordination. Intensive Care Med, 1994;20:225-232

04. Redding GJ - Current concepts in adult respiratory distress syndrome in children. Curr Opin Pediatr, 2001;13:261-266.

05. Gattinoni L, Presenti A, Bombino M - Relationships between lung computed tomographic density, gas exchange and PEEP in acute respiratory failure. Anesthesiology, 1988;69:824-832.

06. Kolobow T, Moretti MP, Fumagalli R et al - Severe impairment of lung function induced by high peak airway pressure during mechanical ventilation. An experimental study. Am Rev Respir Dis, 1987;135:312-315.

07. Hickling KG, Henderson SJ, Jackson R - Low mortality associated with low volume pressure limited ventilation with permissive hypercapnia in severe adult respiratory distress syndrome. Intensive Care Med, 1990;16:372-377

08. Dreyfuss D, Soler P, Saumon G - Mechanical ventilation-induced pulmonary edema. Interaction with previous lung alterations. Am J Respir Crit Care Med, 1995;151:1568-1575.

09. Hemmila MR, Napolitano LM - Severe respiratory failure: advanced treatment options. Crit Care Med, 2006;34:(Suppl9):S278-S290.

10. Ranieri VM, Giunta F, Suter PM et al - Mechanical ventilation as a mediator of multisystem organ failure in acute respiratory distress syndrome. JAMA, 2000;284:43-44

11. Marini JJ, Gattinoni L - Ventilatory management of acute respiratory distress syndrome: a consensus of two. Crit Care Med, 2004;32:250-255.

12. Gattinoni L, Caironi P, Carlesso E - How to ventilate patients with acute lung injury and acute respiratory distress syndrome. Curr Opin Crit Care, 2005;11:69-76.

13. Tremblay LN, Slutsky AS - Ventilator-induced lung injury: from the bench to the bedside. Intensive Care Med, 2006;32:24-33.

14. Amato MB, Barbas CS, Medeiros DM et al - Effect of a protective-ventilation strategy on mortality in the acute respiratory distress syndrome. $\mathrm{N}$ Engl J Med, 1998;338:347-354.

15. Ventilation with lower tidal volumes as compared with traditional tidal volumes for acute lung injury and the acute respiratory distress syndrome. The Acute Respiratory Distress Syndrome Network. N Engl J Med, 2000;342:1301-1308.

16. Crotti S, Mascheroni D, Caironi $P$ et al - Recruitment and derecruitment during acute respiratory failure: a clinical study. Am J Respir Crit Care Med, 2001;164:131-140.

17. Richard JC, Maggiore SM, Jonson B et al - Influence of tidal volume on alveolar recruitment. Respective role of PEEP and a recruitment maneuver. Am J Respir Crit Care Med, 2001;163:1609-1613.

18. Radermacher P, Santak P, Becker $\mathrm{H}$ et al - Prostaglandin E1 and nitroglycerin reduce pulmonary capillary pressure but worsen ventilation-perfusion distribution in patients with adult respiratory distress syndrome. Anesthesiology, 1989;70:601-609.
19. Furchgott RF, Zawadzki JV - The obligatory role of endothelial cells in the relaxation of arterial smooth muscle by acetylcholine. Nature, 1980;288:373-376.

20. Szabo C - Alterations in Nitric Oxide Production in Various Forms of Circulatory Shock, em: Fink MP - New Horizons. The Science and Practice of Acute Medicine. Baltimore (MD): Williams \& Wilkins, 1995;3:2-32.

21. Germain JF, Mercier JC, Casadevall I et al - Is there a role for inhaled nitric oxide in pediatric ARDS? Pediatr Pulmonol Suppl, 1995;11:110112.

22. Troncy E, Francoeur M, Blaise G - Inhaled nitric oxide: clinical applications, indications, and toxicology. Can J Anaesth, 1997;44:973-988.

23. Rossaint R, Falke KJ, Lopez F et al - Inhaled nitric oxide for adult respiratory distress syndrome. N Engl J Med, 1993;328:399-405.

24. Abman SH, Griebel JL, Parker DK et al - Acute effects of inhaled nitric oxide in children with severe hypoxemic respiratory failure. J Pediatr, 1994;124:881-888.

25. Lotti GA, Olivei MC, Palo A et al - Acute effects of inhaled nitric oxide in adult respiratory distress syndrome. Eu Respir J, 1998;12:1164-1171.

26. Troncy E, Collet JP, Shapiro S et al - Inhaled nitric oxide in acute respiratory distress syndrome: a pilot randomized controlled study. Am J Respir Crit Care Med, 1998;157:1483-1488.

27. Okamoto K, Hamaguchi M, Kukita I et al - Efficacy of inhaled nitric oxide in children with ARDS. Chest, 1998;114:827-833.

28. Ream RS, Hauver JF, Lynch RE et al - Low-dose inhaled nitric oxide improves the oxygenation and ventilation of infants and children with acute, hypoxemic respiratory failure. Crit Care Med, 1999;27:989-996.

29. Dobyns EL, Cornfield DN, Anas NG et al - Multicenter randomized controlled trial of the effects of inhaled nitric oxide therapy on gas exchange in children with acute hypoxemic respiratory failure. J Pediatr, 1999;134:406-412.

30. Razavi HM, Werhun R, Scott JA et al - Effects of inhaled nitric oxide in a mouse model of sepsis-induced acute lung injury. Crit Care Med, 2002;30:868-873.

31. Fioretto JR, Bonatto RC, Ricchetti SM et al - Early administration of inhaled nitric oxide to children with acute respiratory distress syndrome and its effects on oxygenation and ventilator settings: prospective preliminary report of ten patients. Croat Med J, 2001;42:527-534.

32. Sokol J, Jacobs SE, Bohn D - Inhaled nitric oxide for acute hypoxemic respiratory failure in children and adults. Cochrane Database Syst Rev, 2006;(3):CD002787.

33. Fioretto JR, de Moraes MA, Bonatto RC et al - Acute and sustained effects of early administration of inhaled nitric oxide to children with acute respiratory distress syndrome. Pediatr Crit Care Med, 2004;5:469474.

34. Kinsella JP, Truog WE, Walsh WF - Randomized, multicenter trial of inhaled nitric oxide and high-frequency oscillatory ventilation in severe, persistent pulmonary hypertension of the newborn. J Pediatr, 1997;131:5562.

35. Putensen C, Rasanen J, Lopez FA et al - Continuous positive airway pressure modulates effect of inhaled nitric oxide on the ventilation-perfusion distributions in canine lung injury. Chest, 1994;106:1563-1569.

36. Cuthbertson $\mathrm{BH}$, Dellinger $\mathrm{P}$, Dyar OJ et al - UK guidelines for the use of inhaled nitric oxide therapy in adult ICUs. Intensive Care Med, 1997;23:1212-1218.

37. Fioretto JR - Inhaled nitric oxide in pediatrics. J Pediatr, 2003;79:(Suppl2):S177-S186.

38. Fioretto JR - Óxido nítrico inalatório na síndrome do desconforto respiratório agudo. Rev Paul Ped, 2000;18:201-204.

39. Johannigman JA, Davis K Jr, Miller SL et al - Prone positioning and inhaled nitric oxide: synergic therapies for acute respiratory distress syndrome. J Trauma, 2001;50:589-596.

40. Mehta S, MacDonald R, Hallett DC et al - Acute oxygenation response to inhaled nitric oxide when combined with high-frequency oscillatory ventilation in adults with acute respiratory distress syndrome. Crit Care Med, 2003;31:383-389.

41. Breuer J, Waidelich F, Irtel von Brenndorff C et al - Technical considerations for inhaled nitric oxide therapy: time response to nitric oxide dosing changes and formation of nitric dioxide. Eur J Pediatr, 1997;156:460-462.

42. Gianetti J, Bevilacqua S, De Caterina R - Inhaled nitric oxide: more than a selective pulmonary vasodilator. Eur J Clin Invest, 2002;32:628-635. 